\title{
Multicontrast MR Imaging at 7T in Multiple Sclerosis: Highest Lesion Detection in Cortical Gray Matter with 3D-FLAIR
}

\author{
I.D. Kilsdonk, W.L. de Graaf, A. Lopez Soriano, J.J. Zwanenburg, F. Visser, J.P.A. Kuijer, J.J.G. Geurts,
}

P.J.W. Pouwels, C.H. Polman, J.A. Castelijns, P.R. Luijten, F. Barkhof, and M.P. Wattjes

\begin{abstract}
BACKGROUND AND PURPOSE: 7T MR imaging has led to improved detection and classification of cortical MS lesions, mainly based on T2*-weighted gradient-echo sequences. Depiction of cortical GM by using the recommended MS imaging protocol has not yet been investigated at 7T. We aimed to investigate prospectively which recommended sequence for clinical use has the highest value at 7T, in terms of GM and WM lesion detection.
\end{abstract}

MATERIALS AND METHODS: Thirty-seven patients with MS (mean age, 43.8 years; 25 women) and 7 healthy controls (mean age, 40.4 years; 5 women) underwent multicontrast 7T MR imaging including the recommended clinical 2D-T2WI, 3D-TIWI, 3D-FLAIR, and GMspecific 3D-DIR. Lesions were scored and categorized anatomically by 3 raters, in consensus. The value of sequences was evaluated lesion-wise and patient-wise (Wilcoxon signed-rank test).

RESULTS: At 7T, 3D-FLAIR detected the highest number of total cortical GM lesions (217), 89\% more than 3D-DIR and $87 \%$ and $224 \%$ more than 2D-T2WI and 3D-T7WI. Patient-wise analysis showed that this difference between 3D-FLAIR and 3D-DIR was statistically significant $(P<.04)$, and most pronounced for the number of mixed lesions $(P<.03)$. 3D-FLAIR also detected the highest number of total WM lesions (2605), but the difference with 3D-DIR and 3D-TIWI was not significant.

CONCLUSIONS: When using recommended clinical sequences at 7T, the best way to detect cortical GM lesions is with 3D-FLAIR and not by GM-specific 3D-DIR or by conventional 2D-T2WI and 3D-TIWI sequences.

ABBREVIATIONS: 2D-T2WI = 2D dual-echo T2-weighted; DIR = double inversion recovery; $\mathrm{GM}$ = gray matter; MAGNIMS = Magnetic Imaging in MS; mixed = mixed GM/WM matter

$\mathbf{M}_{\mathrm{r}}^{\mathrm{s}}$ $\mathrm{S}$ has been primarily regarded as a WM disease, which is reflected in current MR imaging and International Panel diagnostic criteria. ${ }^{1}$ However, early histopathologic studies have already recognized the involvement of cortical GM, ${ }^{2}$ and during the past years, GM abnormalities in MS have been increasingly investigated. Histopathologic studies report that up to $60 \%$ of total MS lesions affect the cortical GM. ${ }^{3,4}$ Unfortunately, the sensitivity of conventional MR imaging techniques to detect cortical lesions remains poor compared with histopathologic studies. ${ }^{3,5-7}$

In the clinical setting, it is highly relevant that GM abnormal-

Received May 11, 2012; accepted after revision July 4.

From the Departments of Radiology (I.D.K., W.L.d.G., A.L.S., J.A.C., F.B., M.P.W.), Physics and Medical Technology (J.P.A.K., P.J.W.P.), Anatomy and Neuroscience, Section of Clinical Neuroscience (J.J.G.G.), and Neurology (C.H.P.), VU University Medical Center Amsterdam, the Netherlands; and Department of Radiology (I.J.Z., F.V., P.R.L.), University Medical Center Utrecht, Utrecht, the Netherlands. Please address correspondence to Iris D. Kilsdonk, Department of Radiology, PO Box 7057, 1007 MB Amsterdam, the Netherlands; e-mail: i.kilsdonk@vumc.nl

http://dx.doi.org/10.3174/ajnr.A3289 ities in MS can be visualized in vivo: first, because GM abnormalities strongly explain cognitive and physical disability, ${ }^{8,9}$ and second, because focal GM lesions are rather specific for MS. They already occur in the earliest stages of the disease, ${ }^{10,11}$ and it was suggested that the McDonald criteria for the diagnosis of MS - at present completely based on WM lesion detection ${ }^{1,12}$ — will be of increased accuracy when cortical GM lesions are included. ${ }^{13}$

Due to a higher SNR with increased spatial resolution, MS lesion detection has improved by moving from 1.5T MR imaging to $3 \mathrm{~T} .{ }^{14}$ At both field strengths, a common finding is that FLAIR detects the highest amount of WM lesions and DIR has the highest sensitivity in detecting cortical GM lesions. ${ }^{15,16}$ The introduction of ultra-high-field 7T systems has led to improved detection and classification of cortical lesions in patients with MS, mainly by using experimental $\mathrm{T} 2{ }^{\star}$-weighted gradient-echo sequences. ${ }^{17-20}$ The depiction of cortical GM by using the standard imaging protocol for MS, ${ }^{21}$ including T1WI, T2WI and FLAIR sequences, has not yet been investigated at 7T and neither has the use of DIR at 7T. This is mainly due to the challenging application of 3D FLAIR and DIR sequences on a 7T system, because of, among others, 
Table 1: Sequence parameters per pulse sequence at 7T

\begin{tabular}{|c|c|c|c|c|}
\hline & 3D-DIR & 3D-FLAIR & 2D-T2WI & 3D-TIWI \\
\hline & TSE & TSE & TSE & TFE \\
\hline TR (ms) & 8000 & 8000 & 4969 & 7.0 \\
\hline TE (1/2) (ms) & 294 & 303 & $21 / 80$ & 2.9 \\
\hline $\mathrm{TI}(1 / 2)(\mathrm{ms})$ & $3150 / 550^{a}$ & 2325 & - & 1129 \\
\hline Flip angle $\left({ }^{\circ}\right)$ & 100 & 100 & 90 & 8 \\
\hline Turbo factor (-) & 128 & 125 & 8 & 312 \\
\hline Acquisition resolution (mm) & $1.0 \times 1.0 \times 0.8$ & $0.8 \times 0.8 \times 0.8$ (Sagittal) & $0.7 \times 1.0 \times 2.0($ Axial $)$ & $0.8 \times 0.8 \times 0.8$ (Sagittal) \\
\hline Reconstructed resolution (mm) & $0.5 \times 0.5 \times 0.4$ & $0.49 \times 0.49 \times 0.4$ (Axial) & $0.45 \times 0.45 \times 2.0$ (Axial) & $0.5 \times 0.5 \times 0.4$ \\
\hline Sensitivity encoding & $2.0 \times 3.0(\mathrm{AP} \times \mathrm{RL})^{\mathrm{b}}$ & $2.5 \times 2.5(\mathrm{AP} \times \mathrm{RL})^{\mathrm{b}}$ & $2.1(\mathrm{RL})$ & $2.0(R L)$ \\
\hline Acquisition time (min/sec) & 11:04 & 13:06 & $8: 07$ & $9: 43$ \\
\hline
\end{tabular}

Note:-TFE indicates turbo field echo; AP, anterior-posterior; LR, left-right.

a The long inversion time (TII) is the interval between the first $180^{\circ}$ inversion pulse and the $90^{\circ}$ excitation pulse, and the short inversion time (TI2) is the interval between the second $180^{\circ}$ inversion pulse and the $90^{\circ}$ excitation pulse.

${ }^{\mathrm{b}}$ Sensitivity encoding applied in 2D.

specific absorption rate restrictions. Nonetheless, both sequences have been successfully implemented at 7T recently. ${ }^{22,23}$

The aim of this prospective study was to investigate which sequence has the highest value at 7T in terms of GM and WM lesion detection: recommended sequences for clinical use (2DT2WI, 3D-T1WI, and 3D-FLAIR) or a sequence that was developed to specifically depict the GM of the brain (3D-DIR). The results of this study will be a preliminary finding for this multicontrast protocol at 7T, according to our specific study design and sequence parameters.

\section{MATERIALS AND METHODS \\ Participants}

Thirty-seven patients were prospectively recruited from our neurology outpatient clinic. Inclusion criteria were clinically definite MS according to the 2005 revised International Panel (McDonald) criteria ${ }^{24}$ and age between 18 and 60 years. Exclusion criteria were the presence or a medical history of other neurologic or vascular disorders, recent relapses ( $<3$ months), and standard contraindications for MR imaging (eg, claustrophobia). Next to these standard contraindications, local high-field MR imaging safety regulations also excluded subjects with any (suspected) metal objects in or on the body as a result of medical interventions in the past.

Seven healthy control subjects were also enrolled in this study from February 2009 till June 2011. A small part of the study population (10 patients with MS and 5 healthy controls) has been incorporated in an explorative feasibility study, which was published earlier. $^{22}$

The institutional ethical review board approved the study, and all subjects gave written informed consent before participation.

\section{MR Imaging Acquisition}

All subjects were imaged on a whole-body 7T MR system (Achieva; Philips Healthcare, Best, the Netherlands), with a slew rate of $200 \mathrm{~T} / \mathrm{m} / \mathrm{s}$, maximum gradient strength of $40 \mathrm{mT} / \mathrm{m}$, by using a 16-channel phased array head coil (Nova Medical, Wilmington, Massachusetts). The protocol included the following pulse sequences: axial 2D-T2WI, sagittal 3D-T1WI, sagittal 3DFLAIR, and sagittal 3D-DIR. The 3D-FLAIR and the 3D-DIR sequences both used magnetization preparation to reduce unwanted T1-weighting. ${ }^{23}$ Detailed sequence parameters are given in Table 1.
Preceding image analysis, all sagittal 3D images were reconstructed in the axial plane corresponding to the 2D-T2WI, with the same section thickness, by using the same repositioning.

\section{Image Analysis}

Images were interpreted by 3 raters in consensus: I.D.K. and W.L.d.G. (PhD students with 2 and 5 years' experience in MR image reading, respectively) and A.L.S. (neuroradiologist, 7 years' experience). During scoring, images of pulse sequences per subject were separated and presented in random order, to avoid recall bias. Raters were blinded to patient identification and clinical or paraclinical information.

Before the evaluation of lesions, the quality of images (artifacts and image homogeneity) was assessed for each pulse sequence. Contrast ratios of the sequences are reported elsewhere. ${ }^{22}$ For each subject and pulse sequence, lesions were counted and categorized according to their anatomic location: periventricular WM lesions in contact with the ventricles; deep white matter lesions not in contact with ventricles or cortex; juxtacortical WM lesions in contact with the cortex; mixed lesions located in the GM as well as in the WM; intracortical lesions located completely within the cortical GM. Only supratentorial WM and cortical GM were included in the analysis because the sensitivity of the coil did not cover infratentorial regions in all subjects. Besides specific anatomic locations, we defined combined regions: total WM (periventricular + deep white matter + juxtacortical), total cortical GM (mixed + intracortical), and total lesion number (total $\mathrm{WM}+$ total GM). Focal areas of hypointense (3D-T1WI) or hyperintense (2D-T2WI, 3D-FLAIR, and 3D-DIR images) signal intensity compared with the surrounding WM and GM, with a minimum size of 3 voxels, were classified as lesions. Scoring of cortical GM lesions was performed by using consensus guidelines developed by the MAGNIMS study group regarding DIR sequences (though these were not primarily designed for $7 \mathrm{~T}$ scorings). ${ }^{25}$

Lesions were marked by a scoring tool developed in-house, which was used as a plug-in in Medical Image Processing, Analysis, and Visualization software (Version 5.1.1, National Institutes of Health, Bethesda, Maryland; http://mipav.cit.nih.gov). Results per subject were collected by a Matlab script (Version 7.1; MathWorks, Natick, Massachusetts) written in-house. After analysis of the results, we reviewed a subset of patients and compared 3DFLAIR and 3D-DIR side-by-side to explain our results, mostly 
because they conflicted with earlier studies at lower (1.5 and 3T) field strengths.

\section{Statistical Analysis}

For each subject, the number of lesions per anatomic location and sequence was assessed and analyzed by the Statistical Package for the Social Sciences, Version 15.0 (SPSS, Chicago, Illinois). In a lesion-wise analysis, the total number of lesions per anatomic region for each sequence was presented, as well as the mean lesion count per patient. Results were compared patient-wise by a Wilcoxon signed-rank test because the total lesion number showed a non-normal distribution that could not be transformed into normality. Results from the pair-wise comparison were Bonferronicorrected for multiple (6 pair-wise) comparisons. Corrected $P$ values $\leq .05$ were considered statistically significant.

\section{RESULTS}

\section{Demographic Data}

Thirty-seven patients with MS (25 women, 12 men) and 7 healthy controls ( 5 women, 2 men) were included in the study. The mean age of the patients with MS at the time of MR imaging was $43.8 \pm$ 8.3 years, and the median Expanded Disability Status Scale score ${ }^{26}$ was 4 (range, $0-7.5$ ). Twenty-two patients had relapsing-remitting MS, 9 had primary-progressive MS, and 6 had secondaryprogressive MS. The mean age of the healthy controls was $40.4 \pm$ 8.9 years.

\section{Healthy Control Subjects}

In the healthy control subjects, 40 lesions were found on the 3DFLAIR images, 26 on 3D-DIR, 24 on 2D-T2WI, and 21 on 3DT1WI. Of these lesions, $79 \%$ were periventricular and deep white matter lesions, presumably of vascular ischemic origin. No intracortical lesions were identified in any of the healthy control subjects; only 1 mixed lesion was found in 1 healthy control on 3DDIR. An example of the 7T sequences in a healthy control subject can be found in Fig 1.

\section{Patients with MS: Lesion-Wise Analysis}

Overall, the 3D-FLAIR sequence detected the highest number of lesions compared with other sequences. In total GM, 217 lesions were detected at 3D-FLAIR, which was $89 \%$ more compared with 3D-DIR (115), 87\% more compared with 2D-T2WI (116), and $224 \%$ more compared with 3D-T1WI (67). The difference was the largest for the detection of mixed lesions at 3D-FLAIR (178), with which $147 \%$ more mixed lesions were detected than on 3D-DIR (72), 117\% (82) more than on 2D-T2WI, and 242\% (52) more than on 3D-T1WI. The highest number of purely intracortical lesions was detected at 3D-DIR (43), a 10\% increase compared with 3D-FLAIR (39) and a $27 \%$ and a $187 \%$ increase when compared with 2D-T2WI (34) and 3D-T1WI (15), respectively. Images of intracortical lesion detection with the different $7 \mathrm{~T}$ sequences used in the study are shown in Fig 2.

In WM, 3D-FLAIR also detected the highest number of lesions (2605), a relative gain of 12\% compared with 2D-T2WI (2321); the difference with both 3D-DIR and 3D-T1WI was small: $1 \%$ (2582 and 2583, respectively). In total, 3D-FLAIR detected the highest number of lesions; 2822 lesions meant a relative gain of

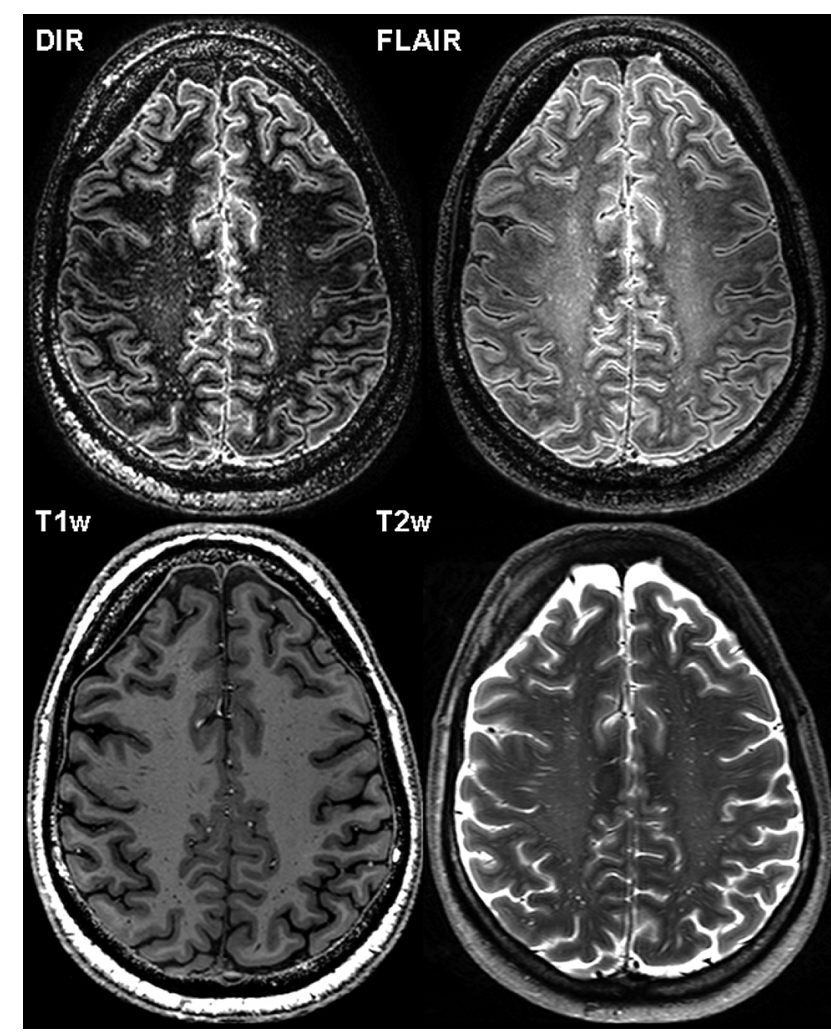

FIG 1. Axial 3D-DIR, 3D-FLAIR, 3D-TIWI, and 2D-T2WI at 7T MR imaging of a 50-year-old healthy male control. Note the hyperintense visualization of both the outer (subpial) layers of the cortex and perivascular spaces on both 3D-DIR and 3D-FLAIR images.

$16 \%$ compared with 2D-T2WI (2437), 7\% compared with 3DT1WI (2650), and 5\% compared with 3D-DIR (2697) images. For more detailed information on lesion counts per anatomic WM region, see Table 2 .

\section{Patients with MS: Patient-Wise Analysis}

In this analysis, every sequence was compared with the 3 other sequences, per patient. In terms of total GM lesion detection, patient-wise analysis showed significantly improved lesion detection at 3D-FLAIR compared with 3D-DIR $(P<.04)$ and 3DT1WI $(P<.01)$ sequences. This was mostly due to a higher detection of mixed lesions at 3D-FLAIR, which was statistically significant compared with 3D-DIR $(P<.03)$ and 3D-T1WI $(P<$ $.01)$.

Concerning the WM, juxtacortical lesion detection at 3D-DIR was significantly higher than in 2D-T2WI $(P<.01)$; this finding also held true for 3D-FLAIR and 3D-T1WI compared with 2DT2WI $(P<.01$ and $P<.01)$. Total WM lesion detection did not differ significantly among all sequences nor did the number of periventricular and deep white matter lesions.

\section{DISCUSSION}

Our results show that 3D-FLAIR detects the highest number of MS lesions at 7T, in total cortical GM and in total WM. On FLAIR images, signal from the CSF is nulled, increasing contrast between lesions and adjacent CSF. At a standard field strength (1.5T), this result meant an increase in MS lesion detection compared with conventional T2WI sequences, especially for juxtacortical le- 


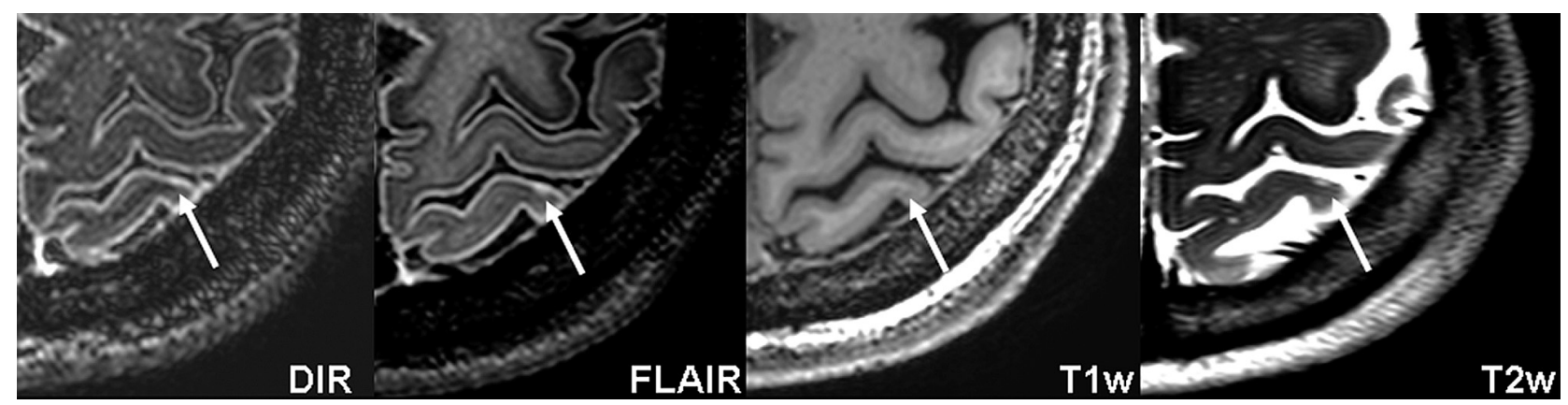

FIG 2. Axial 3D-DIR, 3D-FLAIR, 3D-TIWI, and 2D-T2WI at 7T MR imaging of a 37-year-old female patient with secondary-progressive MS. Arrows indicate an intracortical lesion that was scored on 3D-FLAIR and 2D-T2WI but not on 3D-DIR and 3D-TIWI.

Table 2: Lesion-wise analysis in patients with MS: total lesion detection and mean lesion count per patient ${ }^{\mathrm{a}}$

\begin{tabular}{lcccc}
\hline & 3D-DIR (mean) & 3D-FLAIR (mean) & 2D-T2WI (mean) & 3D-TIWI (mean) \\
\hline PV & $692(18.7 \pm 16.5)$ & $594(16.1 \pm 13.5)$ & $584(15.8 \pm 16.8)$ & $621(16.8 \pm 16.7)$ \\
DWM & $1162(31.4 \pm 32.3)$ & $1197(32.4 \pm 27.3)$ & $1323(35.8 \pm 34.2)$ & $1109(30.0 \pm 34.6)$ \\
JC & $728(19.7 \pm 29.7)$ & $814(22.0 \pm 29.4)$ & $414(11.2 \pm 16.8)$ & $853(23.1 \pm 30.1)$ \\
Total WM & $2582(69.8 \pm 7.1)$ & $2605(70.4 \pm 8.2)$ & $2321(62.7 \pm 13.1)$ & $2583(69.8 \pm 6.6)$ \\
Mixed & $72(1.9 \pm 3.0)$ & $178(4.8 \pm 7.9)$ & $82(2.2 \pm 3.8)$ & $52(1.4 \pm 1.9)$ \\
IC & $43(1.2 \pm 2.4)$ & $39(1.1 \pm 2.7)$ & $34(0.9 \pm 1.9)$ & $15(0.4 \pm 0.9)$ \\
Total cortical GM & $115(3.1 \pm 0.6)$ & $217(5.9 \pm 2.7)$ & $116(3.1 \pm 0.9)$ & $67(1.8 \pm 0.7)$ \\
Total WM+GM & $2697(72.9 \pm 12.9)$ & $2822(76.3 \pm 12.7)$ & $2437(65.9 \pm 14.1)$ & $2650(71.6 \pm 13.1)$ \\
\hline
\end{tabular}

Note:-DWM indicates deep white matter; IC, intracortical; JC, juxtacortical; PV, periventricular.

${ }^{a}$ Data represent numbers of detected lesions per anatomic region.

sions. ${ }^{15,27,28}$ At 3T, FLAIR showed an increased lesion detection compared with $1.5 \mathrm{~T}$, and it showed superiority over conventional T2WI and T1WI sequences for WM lesion detection. ${ }^{29,30}$ Regarding cortical GM lesions at lower field strengths, the highest lesion detection was found by DIR, which at $1.5 \mathrm{~T}$ and at $3 \mathrm{~T}$, suppresses the signal of both CSF and WM, improving the visibility of the

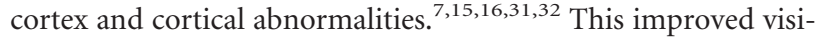
bility of cortical abnormalities at DIR did not hold in our 7T dataset. The detection of total GM lesions was higher on 3D-FLAIR than on 3D-DIR, mostly due to a considerably superior detection of mixed lesions. The higher total GM and mixed-lesion detection at 3D-FLAIR was statistically significant when tested in a patient-wise analysis. Although the detection of purely intracortical lesions was higher at 3D-DIR compared with the other sequences, this did not reach statistical significance in the patient-wise comparison. The $10 \%$ gain in purely intracortical lesion detection on 3D-DIR compared with 3D-FLAIR concerned only 4 lesions.

In the present literature, there are several studies that focus on the detection of cortical GM lesions at 7T, ${ }^{17-19,33,34}$ mostly reporting that $\mathrm{T} 2{ }^{\star}$-weighted gradient-echo sequences improve detection. One of these studies by using $\mathrm{T} 2{ }^{\star}$-weighted gradient-echo images even suggested that this sequence should be the new criterion standard for the detection of cortical lesions in patients with MS. ${ }^{33}$ Another recent study reported that white matter signal attenuation at $7 \mathrm{~T}$ is feasible and is able to detect cortical abnormalities at 7T. ${ }^{35}$ To our knowledge, the value of the recommended sequences for clinical use at 7T has not yet been investigated nor has the use of DIR. Our results show that the highest total cortical lesion detection at 7T is gained with 3D-FLAIR. Future studies should compare the difference between these experimental $\mathrm{T} 2{ }^{*}$ weighted gradient-echo or white matter-attenuated sequences and clinical 3D-FLAIR sequences and elucidate which sequence has the highest sensitivity in terms of 7T cortical lesion detection.
At even a higher field strength (9.4T), T2WI sequences were able to discriminate demyelinated and remyelinated areas in postmortem MS lesions. ${ }^{36,37}$

The results of our 7T study are promising because in vivo imaging of cortical abnormalities in MS has high clinical relevance. Cortical lesions correlate with cognitive impairment ${ }^{8,9}$ and could be used as an outcome measure in MS research or could be of help in the development and monitoring of treatment. Furthermore, the detection of GM abnormalities has prognostic relevance and will help to identify patients with clinically isolated syndrome who will eventually convert to clinically definite MS. It has been proposed that the sensitivity of the criteria for the diagnosis of MS will increase when GM lesions are included. ${ }^{13}$ These McDonald criteria are at present based solely on WM abnormalities, but GM abnormalities might be introduced in the criteria in the future. 7T MR imaging of cortical lesions can play a role in these different aspects and can be of additional value in the care of patients with MS. However, only a limited number of 7T scanners are available, worldwide around $407 \mathrm{~T}$ systems, as mentioned in a recent review on $7 \mathrm{~T}$ MR imaging. ${ }^{38}$

As opposed to 1.5T and 3T, the highest total cortical GM lesion detection at $7 \mathrm{~T}$ was found by the 3D-FLAIR sequence. To investigate this finding further, we reviewed a subset of patients and compared 3D-FLAIR and 3D-DIR sequences side by side (Fig 3). This comparison suggested several possible explanations involving the design of both sequences as well as our rating process. First, the design of sequences on our 7T system causes a multiplelayered appearance of the cortex, which differs between 3D-DIR and $3 \mathrm{D}$-FLAIR ${ }^{22}$ and has recently been described as differing among anatomic regions in the brain. ${ }^{39}$ The cortex is often relatively more hyperintense on 3D-DIR than on 3D-FLAIR because of the attenuation of WM at DIR (Fig 3). This might have caused diminished visibility of cortical lesions on 3D-DIR. Furthermore, 


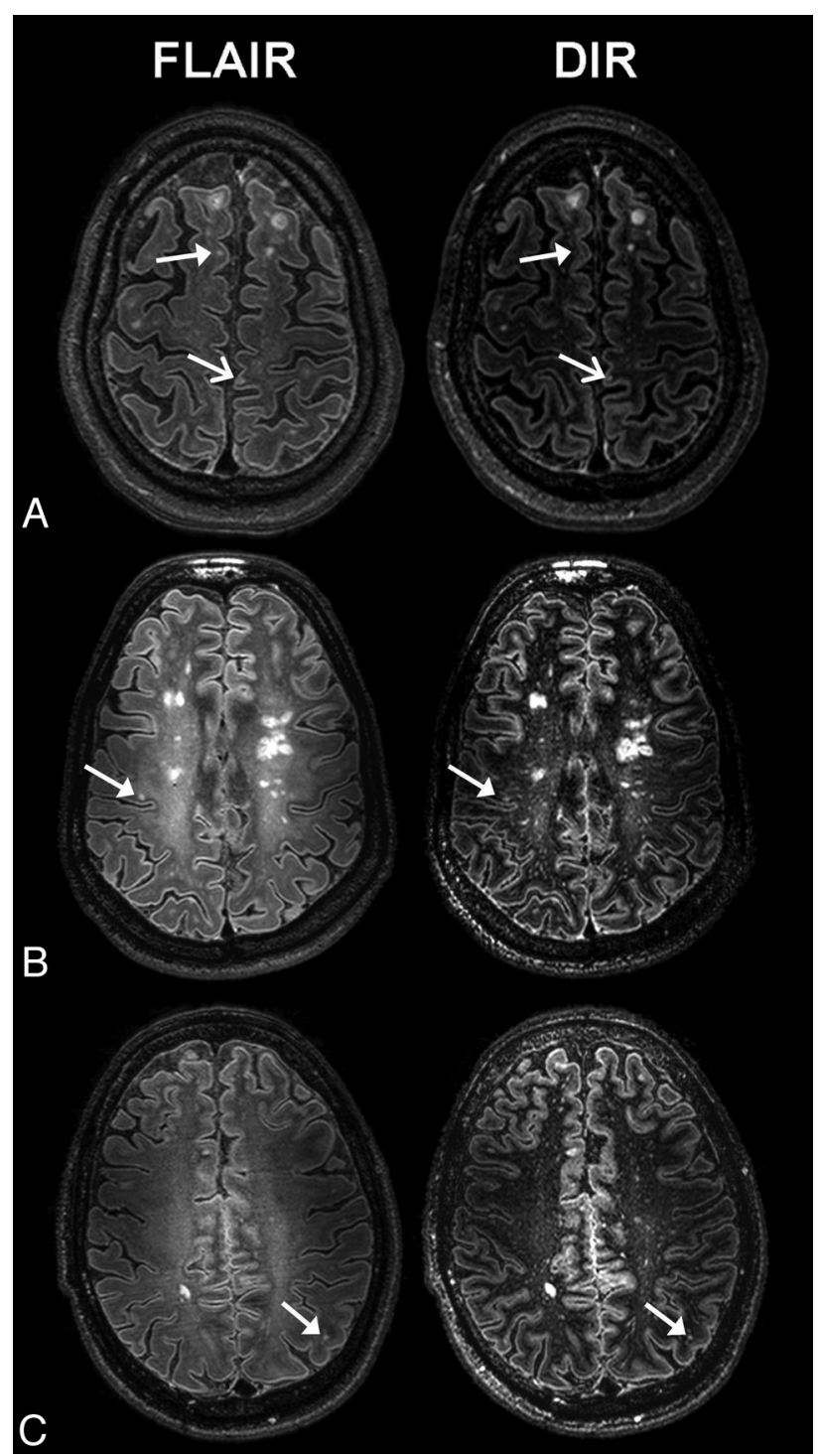

FIG 3. Examples of scoring differences at $7 T$ MR imaging: 3D-FLAIR versus 3D-DIR, found while reviewing a subset of images retrospectively. A, A juxtacortical lesion (closed arrows) that was scored on 3D-FLAIR but not on 3D-DIR because of differences in contrast between the lesion and the cortical gray matter. On 3D-DIR, the cortex looks relatively hyperintense compared with 3D-FLAIR because of the attenuation of white matter, which might have hindered lesion detection. The open arrows show a juxtacortical lesion that was detected at both sequences. $B$, The closed arrows show a mixed lesion that was scored on 3D-FLAIR but not on 3D-DIR because of conservative scoring/distraction by many small hyperintensities and its smaller size on 3D-DIR. C, A juxtacortical lesion that was scored on 3D-FLAIR but not on 3D-DIR images (closed arrows) because tissue was attenuated too much by using the DIR sequence, which decreases the size of the lesion.

due to the combination of 2 inversion pulses at 3D-DIR, besides CSF and WM, additional tissue components are also partly attenuated, including lesions and particularly the periphery of a lesion. This did not make small lesions completely invisible but often too inconspicuous to score, a phenomenon that has also been described at lower field strengths. ${ }^{15}$ Because T1 values are fieldstrength-dependent, this effect could be different at a high field and should be further investigated. In addition to these aspects, the resolution of 3D-FLAIR was better than that of 3D-DIR, 0.51 $\mathrm{mm}^{3}$ versus $0.64 \mathrm{~mm}^{3}$ voxel volumes respectively, which might affect the ability to detect small lesions.

Second, the scoring process might also have hampered lesion detection at 3D-DIR. There is an abundance of small signal abnormalities, mostly perivascular spaces, on 3D-DIR (Fig 1). This may have caused too much distraction while scoring lesions so that they were overlooked or not specified as being a lesion. Furthermore, we used a rather conservative scoring system: Focal hyperintensities were only specified as lesions if they were in line with the consensus guidelines for scoring of cortical lesions on DIR, which were developed by the MAGNIMS study group. ${ }^{25}$ Hyperintensities or inhomogeneities in the cortex were interpreted as artifacts or as small vessels more easily on the 3D-DIR sequence than on the 3D-FLAIR sequence, which may have caused an under-representation of lesions at 3D-DIR.

Contrast ratios of our 7T 3D-FLAIR have been reported to be lower compared with 3D-DIR: GM/WM of 0.40 and 0.35 in healthy controls and patients respectively for FLAIR, compared with 0.93 and 0.87 for DIR. Lesion/WM contrast in FLAIR was 0.86 , whereas it was 2.92 in DIR; lesion/GM was 0.91 in FLAIR and 1.40 in DIR. ${ }^{22}$ Hence contrast ratios are unlikely to have advanced lesion detection at 3D-FLAIR. Despite the higher relative contrast in DIR, the absolute contrast in terms of contrast-to-noise ratios could be less, due to the overall reduction in SNR by the extra inversion pulse.

The lower lesion counts detected by the 3D-DIR sequence cannot be explained by a reclassification phenomenon (ie, the shift from one anatomic region to another because of improved visibility of the cortex). This result was the case at $1.5 \mathrm{~T}$ and 3T DIR, when a slightly lower sensitivity in juxtacortical lesion detection was counterbalanced by an increased detection of mixed or purely intracortical lesions. ${ }^{16,31,32}$ However at $7 \mathrm{~T}$, with higher spatial resolution and different image contrast, visibility of the cortex was improved at all sequences; hence, a reclassification phenomenon was not evident in our data.

Concerning WM lesion detection at 7T, our results showed statistically significant differences in the juxtacortical region. At 3D-FLAIR, 3D-DIR, and 3D-T1WI, a higher number of juxtacortical lesions were detected compared with 2D-T2WI. Total WM lesion detection was highest at 3D-FLAIR when analyzed lesionwise, though the difference with 3D-DIR and 3D-T1WI was negligible ( $1 \%$ higher). When we tested patient-wise, this increased WM lesion detection of 7T 3D-FLAIR, compared with other sequences, was not statistically significant.

Limitations of the study are that we could have taken more advantage of the multicontrast protocol by scoring lesions one on one on all 4 sequences simultaneously. The goal of this study, however, was to evaluate the performance of the sequences regarding overall lesion counts in various anatomic regions among the different sequences, so we chose to score the sequences separately. The scoring process was done by 3 raters in consensus because of the novelty of 7T MR images. A possible limitation could be that we have no intra- or interobserver comparison. In the future, it would be interesting to study this, possibly in addition to comparing interobserver agreement among different field strengths and among raters of different levels of experience. In addition, most important, the results in this study are specific for our MR imaging system and our sequences with mentioned pa- 
rameters. Whether results can be generalized to other 7T MR systems remains a matter for future research.

\section{CONCLUSIONS}

The results of the present study show that according to our study setup and our sequence designs, with the recommended sequences for clinical use at 7T, the best way to detect focal cortical GM abnormalities is with 3D-FLAIR and not a GM-specific 3D-DIR sequence or conventional 2D-T2WI and 3D-T1WI sequences.

Disclosures: Iris D. Kilsdonk—RELATED: Grant: supported by a grant provided by the Noaber Foundation (Lunteren, the Netherlands). Freddy Visser-UNRELATED: Employment: Philips Healthcare (Best, the Netherlands). Chris H. Polman-RELATED: Grant: MS Research Foundation, the Netherlands, ${ }^{*}$ UNRELATED: Consultancy/Research Support: I have received honoraria/consultation fees/research support from Actelion, Biogen Idec, Bayer Schering, GlaxoSmithKline, Merck Serono, Novartis, TEVA, UCB, and Roche.* Jonas A. Castelijns-RELATED: Grant: Noaber Foundation. Frederik Barkhof-RELATED: Grant: Noaber Foundation, ${ }^{*}$ UNRELATED: Board Membership: GE Healthcare, Sanofi-Aventis, Roche, Consultancy: Novartis, Merck-Serono, Biogen-IDEC, Synthon, Payment for Lectures (including service on Speakers Bureaus): MedScape. *Money paid to the institution.

\section{REFERENCES}

1. Polman CH, Reingold SC, Banwell B, et al. Diagnostic criteria for multiple sclerosis: 2010 revisions to the "McDonald criteria." Ann Neurol 2011;69:292-302

2. Brownell B, Hughes JT. The distribution of plaques in the cerebrum in multiple sclerosis. J Neurol Neurosurg Psychiatr 1962;25:315-20

3. Kidd D, Barkhof F, McConnell R, et al. Cortical lesions in multiple sclerosis. Brain 1999;122 (pt 1):17-26

4. Bø L, Vedeler CA, Nyland HI, et al. Subpial demyelination in the cerebral cortex of multiple sclerosis patients. J Neuropathol Exp Neurol 2003;62:723-32

5. Geurts JJG, Bö L, Pouwels PJW, et al. Cortical lesions in multiple sclerosis: combined postmortem MR imaging and histopathology. AJNR Am J Neuroradiol 2005;26:572-77

6. Seewann A, Vrenken H, Kooi E, et al. Imaging the tip of the iceberg: visualization of cortical lesions in multiple sclerosis. Mult Scler 2011;17:1202-10

7. Seewann A, Kooi EJ, Roosendaal SD, et al. Postmortem verification of MS cortical lesion detection with 3D DIR. Neurology 2012;78:302-08

8. Roosendaal SD, Moraal B, Pouwels PJW, et al. Accumulation of cortical lesions in MS: relation with cognitive impairment. Mult Scler 2009; 15:708-14

9. Kutzelnigg A, Lassmann H. Cortical demyelination in multiple sclerosis: a substrate for cognitive deficits? J Neurol Sci 2006;245:123-26

10. Calabrese M, Gallo P. Magnetic resonance evidence of cortical onset of multiple sclerosis. Mult Scler 2009;15:933-41

11. Lucchinetti CF, Popescu BF, Bunyan RF, et al. Inflammatory cortical demyelination in early multiple sclerosis. $N$ Engl J Med 2011;365: 2188-97

12. Montalban X, Tintoré M, Swanton J, et al. MRI criteria for MS in patients with clinically isolated syndromes. Neurology 2010;74:427-34

13. Filippi M, Rocca MA, Calabrese M, et al. Intracortical lesions: relevance for new MRI diagnostic criteria for multiple sclerosis. $\mathrm{Neu}$ rology 2010;75:1988-94

14. Wattjes MP, Barkhof F. High field MRI in the diagnosis of multiple sclerosis: high field-high yield? Neuroradiology 2009;51:279-92

15. Moraal B, Roosendaal SD, Pouwels PJW, et al. Multi-contrast, isotropic, single-slab 3D MR imaging in multiple sclerosis. Eur Radiol 2008;18:2311-20

16. Wattjes MP, Lutterbey GG, Gieseke J, et al. Double inversion recovery brain imaging at $3 \mathrm{~T}$ : diagnostic value in the detection of multiple sclerosis lesions. AJNR Am J Neuroradiol 2007;28:54-59

17. Kollia K, Maderwald S, Putzki N, et al. First clinical study on ultrahigh-field MR imaging in patients with multiple sclerosis: comparison of 1.5T and 7T. AJNR Am J Neuroradiol 2009;30:699-702
18. Mainero $\mathrm{C}$, Benner $\mathrm{T}$, Radding $\mathrm{A}$, et al. In vivo imaging of cortical pathology in multiple sclerosis using ultra-high field MRI. Neurology 2009;73:941-48

19. Pitt D, Boster A, Pei W, et al. Imaging cortical lesions in multiple sclerosis with ultra-high-field magnetic resonance imaging. Arch Neurol 2010;67:812-18

20. Kilsdonk ID, de Graaf WL, Barkhof F, et al. Inflammation high-field magnetic resonance imaging. Neuroimaging Clin N Am 2012;22: 135-57, ix

21. Simon JH, Li D, Traboulsee A, et al. Standardized MR imaging protocol for multiple sclerosis: Consortium of MS Centers consensus guidelines. AJNR Am J Neuroradiol 2006;27:455-61

22. de GraafWL, Zwanenburg JJ, Visser F, et al. Lesion detection at seven Tesla in multiple sclerosis using magnetisation prepared 3D-FLAIR and 3D-DIR. Eur Radiol 2012;22:221-31

23. Visser F, Zwanenburg JJM, Hoogduin JM, et al. High-resolution magnetization-prepared 3D-FLAIR imaging at 7.0 Tesla. Magn Reson Med 2010;64:194-202

24. Polman CH, Reingold SC, Edan G, et al. Diagnostic criteria for multiple sclerosis: 2005 revisions to the McDonald criteria. Ann Neurol 2005;58:840-46

25. Geurts JJ, Roosendaal SD, Calabrese M, et al. Consensus recommendations for MS cortical lesion scoring using double inversion recovery MRI. Neurology 2011;76:418-24

26. Kurtzke J. Rating neurologic impairment in multiple sclerosis: an expanded disability status scale (EDSS). Neurology 1983;33:1444-52

27. Tan IL, Pouwels PJ, van Schijndel RA, et al. Isotropic 3D fast FLAIR imaging of the brain in multiple sclerosis patients: initial experience. Eur Radiol 2002;12:559-67

28. Filippi M, Yousry T, Baratti C, et al. Quantitative assessment of MRI lesion load in multiple sclerosis: a comparison of conventional spin-echo with fast fluid-attenuated inversion recovery. Brain 1996; 119 (pt 4):1349-55

29. Bachmann R, Reilmann R, Schwindt W, et al. FLAIR imaging for multiple sclerosis: a comparative MR study at 1.5 and 3.0 Tesla. Eur Radiol 2006;16:915-21

30. Wattjes MP, Lutterbey GG, Harzheim M, et al. Higher sensitivity in the detection of inflammatory brain lesions in patients with clinically isolated syndromes suggestive of multiple sclerosis using high field MRI: an intraindividual comparison of $1.5 \mathrm{~T}$ with $3.0 \mathrm{~T}$. Eur Radiol 2006;16:2067-73

31. Geurts JJG, Pouwels PJ, Uitdehaag BM, et al. Intracortical lesions in multiple sclerosis: improved detection with 3D double inversionrecovery MR imaging. Radiology 2005;236:254-60

32. Simon B, Schmidt S, Lukas C, et al. Improved in vivo detection of cortical lesions in multiple sclerosis using double inversion recovery MR imaging at 3 Tesla. Eur Radiol 2010;20:1675-83

33. Tallantyre EC, Morgan PS, Dixon JE, et al. 3 Tesla and 7 Tesla MRI of multiple sclerosis cortical lesions. J Magn Reson Imaging 2010;32: 971-77

34. Nielsen AS, Kinkel RP, Tinelli E, et al. Focal cortical lesion detection in multiple sclerosis: 3 Tesla DIR versus 7 Tesla FLASH-T2*. J Magn Reson Imaging 2012;35:537-42

35. Bluestein KT, Pitt D, Sammet S, et al. Detecting cortical lesions in multiple sclerosis at $7 \mathrm{~T}$ using white matter signal attenuation. Magn Reson Imaging 2012;30:907-15

36. Schmierer K, Parkes HG, So P-W. Direct visualization of remyelination in multiple sclerosis using T2-weighted high-field MRI. Neurology 2009;72:472

37. Schmierer K, Parkes HG, So P-W, et al. High field (9.4 Tesla) magnetic resonance imaging of cortical grey matter lesions in multiple sclerosis. Brain 2010;133(pt 3):858-67

38. Moser E, Stahlberg F, Ladd ME, et al. 7-T MR-from research to clinical applications? NMR Biomed 2012;25:695-716

39. Zwanenburg JJ, Hendrikse J, Luijten PR. Generalized multiple-layer appearance of the cerebral cortex with 3D FLAIR 7.0-T MR imaging. Radiology 2012;262:995-1001 